\title{
On the identification of Wiener-Hopf factors*
}

\author{
N. Bayer \\ CWI, P.O. Box 94079, 1090 GB Amsterdam, The Netherlands
}

Received 5 October 1995; revised 12 July 1996

\begin{abstract}
This note is concerned with the identification of the Wiener-Hopf factors of a function $1-f$, where $f$ generates an aperiodic distribution on the integers with a negative mean. The general and rational cases are addressed. We give a concise summary of the main practical facts needed for calculations involving the Wiener-Hopf factors. The basic facts are cited from the literature, but a few aspects are briefly proven here.
\end{abstract}

Keywords: Probability generating function (of a Laurent series form), factorization, quotient factorization, ladder-heights.

\section{Introduction}

Many processes arising in queueing systems are governed by a recurrence equation with the truncation operator $\max \{0, \cdot\}$ or a similar one ${ }^{1}$. The best known example is the waiting time process in a $\mathrm{G} / \mathrm{G} / 1$ queue, which is governed by Lindley's equation. The Wiener-Hopf technique is a useful tool for analyzing such processes. An introduction to the Wiener-Hopf technique, illustrated by examples, can be found in Cohen [5]. A short exposition (limited to Lindley's equation) can also be found in Kleinrock [7, Section 8.2]. Here we are motivated by a class of random walks truncated at zero, arising for example in the study of an M/G/1 queue with negative customers [3]. The technique is used there for calculating a stationary state distribution. But in addition to their usage in calculating stationary distributions, the Wiener-Hopf factors have a key role in the analysis of hitting times and other functionals of trajectories of random walks (Asmussen [1, Chapter VII], Borovkov [4, Chapter 3], Lotov and Khodjibayev [9], Lotov [8]).

To provide a complete solution for a problem approached by the Wiener-Hopf technique, one needs to identify the factors (introduced in the next section) of a function $1-f$, where $f$ is some characteristic function or a probability generating function ${ }^{2}$. We focus here on the case where $f$ is a probability generating function.

\footnotetext{
* Supported by the European grant BRA-QMIPS of CEC DG XIII.

' Such processes also arise in other areas, e.g., inventory systems.

${ }^{2}$ The scope of this note is limited to scalar functions. The need for factorizing a matrix function arises, e.g., in the analysis of processes with Markov-modulated transitions. See de Smit [6] and its bibliographical list.
} 
The treatment of a characteristic function prevails in the literature, and is analogous to a large extent. Note that the characteristic function of an integer-valued random variable with a rational generating function is generally not rational. In the literature there is a frequent use of bivariate functions of the form

$$
\varphi(r, s)=1-r f(s) \text {. }
$$

Here we focus on the univariate function $1-f$, which arises in sheer stationary treatments. Seemingly, knowledge about $\varphi$ is applicable also to $1-f$. Actually, discontinuity phenomena at $r=1$ give rise to some complications, which necessitate a separate and careful examination of $1-f$. Our goal is to identify its factors and their properties, in general and in the rational case. These properties can be relied upon when a problem involving the factorization is solved at a concrete level.

In section 2 we introduce the Wiener-Hopf factors, along with a formal identification thereof. This formal and implicit identification appears to be about all that is available at the level of full generality; yet, it conveys some essential information. In section 3 we address the rational case. Rational generating functions are splendidly amenable to practical treatment, because they offer a simple and explicit identification of the Wiener-Hopf factors. No other assumption, apart from aperiodicity, rationality, and negative mean, is needed.

For more information, a historical survey, and an elaborated bibliography, see Asmussen [2]. Recent results concerning the identification of the Wiener-Hopf factors of Gaussian characteristic functions are given in Lotov [8]. These results are quite involved, but explicit.

\section{The general case}

Assume that $f$, defined on the unit circle $|s|=1$, is of the form $f(s)=\mathbb{E}\left(s^{X}\right)$ with $X$ being some aperiodic integer-valued random variable having a finite negative mean. We are interested in identifying the Wiener-Hopf factors of $1-f$. The WienerHopf factorization identity [1, p. 172] reads

$$
1-f(s)=\left[1-g_{+}(s)\right]\left[1-g_{-}(s)\right], \quad|s|=1,
$$

where $g_{+}$and $g_{-}$are the ladder-height generating functions ${ }^{3}$ associated with $f ; g_{+}$ is the ascending-weak, and $g_{-}$is the descending-strict ${ }^{4}$. The ascending-weak (resp. descending-strict) ladder height takes on nonnegative (resp. negative) values, and thus $g_{+}$(resp. $g_{-}$) is defined on $|s| \leqslant 1$ (resp. $|s| \geqslant 1$ ). Generally, the identification of $1-g_{+}$(or equivalently $1-g_{-}$) is not trivial. However,

\footnotetext{
${ }^{3}$ In [1], characteristic functions take the place of generating functions.

${ }^{4}$ The definition of the ladder-heights, which are functionals of trajectories constructed of samples from $f$, can be found in [1, p. 165]. In [1], the strictness/weakness is reversed. The version used here will turn out to be more informative in the rational case. The two versions can be inferred from each other by considering the same trajectories with a negative sign.
} 
Remark 1 . The case $X \geqslant-1$ occurs in many applications and offers a trivial identification: The descending-strict ladder-height is deterministically -1 , so $g_{-}(s)=s^{-1}$.

The factor $1-g_{+}$is identified in $[1, \text { p. } 177 \text {, equation (4.6) }]^{5}$ in terms of distributions of sums $S_{n}$ of $n$ independent samples from $f$ :

$$
1-g_{+}(s)=\exp \left\{-\sum_{n=1}^{\infty} \frac{1}{n} \mathbb{E}\left(s^{\boldsymbol{S}_{n}} 1_{\left\{\boldsymbol{S}_{n} \geqslant 0\right\}}\right)\right\}, \quad|s| \leqslant 1 .
$$

A similar identification of $1-g_{-}$, valid on $|s| \geqslant 1$, is obtained by replacing the event $\left\{\boldsymbol{S}_{n} \geqslant 0\right\}$ with the complementary event $\left\{\boldsymbol{S}_{n}<0\right\}$; however, the analogy between $1-g_{+}$and $1-g_{-}$is not complete, affecting the validation of the latter. In contrast to the series in equation (3), the series with the complementary events fails to converge at $s=1$. The convergence is relied upon in the validation of equation (3) ${ }^{6}$. Thus, the identification of $1-g_{-}$must be substantiated separately, by verifying that the expression is analytical in $|s| \geqslant 1$ and satisfies equation (2). The divergence of the series with the replaced events at $s=1$ also hides the properties and structure of $1-g_{-}$. The following equivalent formula contains an exponential of a Laurent series which converges throughout $|s| \geqslant 1$, and emphasizes the fact that $1-g_{-}$has a zero at $s=1$ :

$$
1-g_{-}(s)=\frac{s-1}{s} \exp \left\{\sum_{n=1}^{\infty} \frac{1}{n}\left[s^{-n}-\mathbb{E}\left(s^{\boldsymbol{S}_{n}} 1_{\left\{\boldsymbol{S}_{n}<0\right\}}\right)\right]\right\}, \quad|s| \geqslant 1 .
$$

Proof of equation (4). We only need to verify that the expression at the right hand side satisfies equation (2): The convergence of the series inside the exponent at $s=1$ will be indicated in the course of this verification, and the convergence on the rest of the unit circle will follow as a by-product. This last statement holds because $|f(s)|<1$ for every $s \neq 1$ on the unit circle due to the aperiodicity hypothesis, so $1-f(s)$ cannot be zero or infinity. The series has a Laurent form $\sum_{n=1}^{\infty} a_{n} s^{-n}$, which will imply its own convergence and the analyticity of the whole expression in the domain of definition $|s| \geqslant 1$.

Consider then the product of the expressions at the right hand sides of equations (3) and (4). For $s=1$ the series in equation (4) becomes $\sum_{n=1}^{\infty} \frac{1}{n} \mathbb{P}\left\{\boldsymbol{S}_{n} \geqslant 0\right\}$, which is the same convergent series occurring in equation (3). The product thus takes the value 0 , which qualifies as $1-f(1)$. For $|s|=1, s \neq 1$ the product is

$$
\frac{s-1}{s} \cdot \frac{\exp \left\{-\sum_{n=1}^{\infty} \frac{1}{n} \mathbb{E}\left(s^{S_{n}}\right)\right\}}{\exp \left\{-\sum_{n=1}^{\infty} \frac{1}{n}\left(\frac{1}{s}\right)^{n}\right\}}
$$

${ }^{5}$ Corollary 4.3 (p. 175) is also needed, to reverse the strictness/weakness.

${ }^{6}$ In [1], part (iv) of Corollary 4.4 (p. 176) is used in the proof of equation (4.6) (p. 177). 
Remembering that $\mathbb{E}\left(s^{S_{n}}\right)=[f(s)]^{n}$ and that $|f(s)|<1$, and applying the identity

$$
\exp \left[-\sum_{n=1}^{\infty} \frac{1}{n} x^{n}\right]=1-x, \quad|x| \leqslant 1, x \neq 1
$$

we see that (5) is $1-f(s)$.

\subsection{Quotient factorizations} the form

In a stationary treatment, one may be interested in a quotient factorization of

$$
1-f(s)=(s-1) \frac{h_{\text {in }}(s)}{h_{\text {out }}(s)}, \quad|s|=1,
$$

where $h_{\text {in }}$ and $h_{\text {out }}$ are defined and analytical inside $(|s| \leqslant 1)$ and outside $(|s| \geqslant 1)$ the unit circle, respectively. Equations $(3,4)$ show that such a factorization is provided by

$$
h_{\text {in }}=1-g_{+}, \quad h_{\text {out }}=\frac{s-1}{1-g_{-}} .
$$

We see that this $h_{\text {out }}$ has the property $h_{\text {out }}(s) / s \rightarrow 1$ as $s \rightarrow \infty$. The following proposition shows that the quotient factorization defined by equation (7) is in some sense minimal.

Proposition 1. There exist no $h_{\text {in }}$ and $h_{\text {out }}$, analytical in $|s| \leqslant 1$ and $|s| \geqslant 1$, respectively, and satisfying equation (6), such that $h_{\text {out }}(s)=\mathrm{o}(s)$ as $s \rightarrow \infty$.

Proof (semi-probabilistic). We may consistently assume that there exists a probability space supporting an i.i.d. sequence $\left\{\boldsymbol{X}_{j}\right\}$ sampled from $f$, as well as a stationary sequence $\left\{\boldsymbol{Y}_{j}\right\}$ satisfying

$$
\boldsymbol{Y}_{j+1}=\max \left\{0, \boldsymbol{Y}_{j}+\boldsymbol{X}_{j}\right\}, \quad j=0, \pm 1, \ldots .
$$

Suppose that $h_{\text {in }}$ and $h_{\text {out }}$ are analytical in $|s| \leqslant 1$ and $|s| \geqslant 1$, respectively, and satisfy equation (6). A calculation shows ${ }^{7}$ that

$$
h_{\text {in }}(s) \mathbb{E}\left(s^{Y_{1}}\right)=h_{\text {out }}(s) \frac{\mathbb{E}\left(s^{\min \left\{0, Y_{1}+X_{1}\right\}}\right)-1}{s-1}, \quad|s|=1 .
$$

The left and right hand sides of equation (8) can be continued analytically into the domains $|s|<1$ and $|s|>1$, respectively. Therefore there exists some entire function $\psi$ with which they coincide in these domains. If $h_{\text {out }}(s)$ were $\mathrm{o}(s)$, then the right hand side would have been declining to zero as $s \rightarrow \infty$. But this would have implied that $\psi \equiv 0$, which is clearly impossible.

\footnotetext{
${ }^{7}$ Details can be found in Bayer and Boxma [3].
} 
Remark 2 (on the role of continuity considerations). The formula (3) for $1-g_{+}$is obtained through a continuity consideration, applying to the factorization of the bivariate function $\varphi$ of equation (1). (The factorization is with respect to $s$, and the continuity is with respect to $r$, at $r=1$.) The factorization of $\varphi$, for every $|r|<1$, is provided by Spitzer's identity, which is recalled in most literature sources on the subject, and on which we do not dwell here. We have noted above that $1-g_{-}$cannot be obtained by the same token. This suggests some form of discontinuity. Indeed, the function $\varphi$ is perfectly continuous in $r$, but its set of zeros on the unit circle (with respect to $s$ ) experiences a discontinuity at $r=1$ : This set is $\varnothing$ for every $|r|<1$, and is $\{1\}$ for $r=1$. The role of the zeros will become more apparent in the next discussion of the rational case.

\section{The rational case}

In the case where $f$ is rational, an explicit and practical identification of $1-g_{+}$ and $1-g_{-}$is available. $1-f$ must have a representation

$$
1-f(s)=C \frac{\prod_{i=1}^{k}\left(s-\alpha_{i}\right)}{\prod_{j=1}^{n}\left(s-\beta_{j}\right)}(s-1),
$$

where $C, k, n, \alpha_{1}, \ldots, \alpha_{k}$ and $\beta_{1}, \ldots, \beta_{n}$ are constants. We assume that this representation is reduced, namely $\left\{\alpha_{1}, \ldots, \alpha_{k}\right\} \cap\left\{\beta_{1}, \ldots, \beta_{n}\right\}=\varnothing$. By the very fact that $f$ is well-defined on the unit circle we have

$$
\left|\beta_{j}\right| \neq 1, \quad j=1, \ldots, n \text {. }
$$

$s=1$ is a single zero of $1-f$, since $f^{\prime}(1)=\mathbb{E}(X)<0$. By this fact, together with the fact that $|f(s)|<1$ on the rest of the unit circle, we also have

$$
\left|\alpha_{i}\right| \neq 1, \quad i=1, \ldots, k \text {. }
$$

Proposition 2 (identification of the Wiener-Hopf factors in the rational case). The following two relations hold:

$$
\begin{aligned}
& 1-g_{+}(s)=C \frac{\prod_{\left\{i /\left|\alpha_{i}\right|>1\right\}}\left(s-\alpha_{i}\right)}{\prod_{\left\{j /\left|\beta_{j}\right|>1\right\}}\left(s-\beta_{j}\right)}, \quad|s| \leqslant 1, \\
& 1-g_{-}(s)=(s-1) \frac{\prod_{\left\{i /\left|\alpha_{i}\right|<1\right\}}\left(s-\alpha_{i}\right)}{\prod_{\left\{j /\left|\beta_{j}\right|<1\right\}}\left(s-\beta_{j}\right)}, \quad|s| \geqslant 1 .
\end{aligned}
$$

$1-g_{-}(s)$ tends to 1 when $s \rightarrow \infty$, as can be seen from equation (4) or simply from the fact that $g$ - generates a distribution on negative integers. Letting $s \rightarrow \infty$ in equation (11), we have 


\section{Corollary 3.}

$$
\left|\left\{i /\left|\alpha_{i}\right|<1\right\}\right|-\left|\left\{j /\left|\beta_{j}\right|<1\right\}\right|=-1 .
$$

Proof of Proposition 2. A pair of functions ( $h_{\text {in }}, h_{\text {out }}$ ), defined and analytical in $|s| \leqslant 1$ and $|s| \geqslant 1$, respectively, will be referred to as a proper quotient factorization of $1-f$ if they satisfy equation (6) and both have no zeros on the unit circle. The minimal integer $d$ such that $\lim _{s \rightarrow \infty} h_{\text {out }}(s) / s^{d}$ is finite will be referred to as the degree of the quotient factorization; $d=-\infty$ cannot occur, $d=+\infty$ is allowed. The pair

$$
\left(1-g_{+}, \frac{s-1}{1-g_{-}}\right)
$$

is a proper quotient factorization, of degree 1 . The pair $\left(h_{\mathrm{in}}^{*}, h_{\mathrm{out}}^{*}\right)$, where

$$
h_{\mathrm{in}}^{*}(s) \triangleq C \frac{\prod_{\left\{i /\left|\alpha_{i}\right|>1\right\}}\left(s-\alpha_{i}\right)}{\prod_{\left\{j /\left|\beta_{j}\right|>1\right\}}\left(s-\beta_{j}\right)}, \quad h_{\mathrm{out}}^{*}(s) \triangleq \frac{\prod_{\left\{j /\left|\beta_{j}\right|<1\right\}}\left(s-\beta_{j}\right)}{\prod_{\left\{i /\left|\alpha_{i}\right|<1\right\}}\left(s-\alpha_{i}\right)},
$$

is also a proper quotient factorization. Showing the equality between the two pairs will prove the proposition. This equality will follow from the minimality of the degree of the factorization (13) (recall Proposition 1), and from

Claim. Any proper quotient factorization of a minimal degree must be equal to $\left(h_{\text {in }}^{*}, h_{\text {out }}^{*}\right)$, up to a multiplicative constant.

The multiplicative constant involved here is 1 , by the calibration

$$
\lim _{s \rightarrow \infty} \frac{1}{s} h_{\text {out }}^{*}(s)=1=\lim _{s \rightarrow \infty} \frac{1}{s} \cdot \frac{s-1}{1-g_{-}} .
$$

Proof of the claim. Let $\left(h_{\mathrm{in}}, h_{\text {out }}\right)$ be an arbitrary proper quotient factorization of a minimal degree. We show that $\left(h_{\text {in }}, h_{\text {out }}\right)$ must in fact be equal to $\left(h_{\text {in }}^{*}, h_{\text {out }}^{*}\right)$, multiplied by some constant $K$. The minimality hypothesis prohibits $h_{\text {in }}$ and $h_{\text {out }}$ from having zeros in the interior of their domains of definition: If $z$ were a zero of $h_{\text {in }}$ in $|s|<1$, or a zero of $h_{\text {out }}$ in $|s|>1$, then we could have decreased the degree by dividing both factors by $s-z$. Zeros on the unit circle are also prohibited, by properness. Denote

$$
a(s) \triangleq C \prod_{i=1}^{k}\left(s-\alpha_{i}\right), \quad b(s) \triangleq \prod_{j=1}^{n}\left(s-\beta_{j}\right) .
$$

We have

The function

$$
\frac{h_{\text {in }}(s)}{h_{\text {out }}(s)}=\frac{a(s)}{b(s)}, \quad|s|=1
$$

$$
h_{\text {in }}(s) \frac{b(s)}{a(s)}, \quad|s|<1, s \notin\left\{\alpha_{1}, \ldots, \alpha_{k}\right\}
$$


thus provides an analytical continuation of $h_{\text {out }}$ to the rest of the complex plane, with the exception of the zeros of $a(s)$ in $|s|<1$. The overall set of zeros of the continued version of $h_{\text {out }}$ is $\left\{\beta_{j} /\left|\beta_{j}\right|<1\right\}$; its overall set of singularities consists of the poles $\left\{\alpha_{i} /\left|\alpha_{i}\right|<1\right\}$ (multiplicity is expressed through repetition). Hence, this continued version of $h_{\text {out }}$ must be of the form

$$
K \frac{\prod_{\left\{j /\left|\beta_{j}\right|<1\right\}}\left(s-\beta_{j}\right)}{\prod_{\left\{i /\left|\alpha_{i}\right|<1\right\}}\left(s-\alpha_{i}\right)}=K h_{\text {out }}^{*}(s),
$$

as required. $h_{\text {in }}(s)$ can be obtained in a similar way, or simply extracted from equation (15).

Remark 3 (direct proof of equation (12), outlined by O.J. Boxma). Instead of inferring equation (12) as a corollary of Proposition 2, it can be proven directly through the argument principle ${ }^{8}$. This proof is essentially standard, but involves a subtlety arising from the fact that $1-f$ has a zero on the unit circle $(s=1)$.

Due to the fact that $f(1)$ and $f^{\prime}(1)$ are both real, with $f(1)>0$ and with $f^{\prime}(1)=\mathbb{E}(\boldsymbol{X})<0$, there exists a neighborhood $|s-1|<\varepsilon$ in which $|f(s)|$ is strictly decreasing along horizontal lines. Let the closed contour

$$
\Gamma=\left\{r(\theta) \mathrm{e}^{\mathrm{i} \theta}, 0 \leqslant \theta<2 \pi\right\}
$$

be defined by patching the unit circle in this neighborhood:

$$
r(\theta) \triangleq \begin{cases}1+\varepsilon-\theta, & 0 \leqslant \theta<\varepsilon, \\ 1, & \varepsilon \leqslant \theta<2 \pi-\varepsilon, \\ 1+\varepsilon-(2 \pi-\theta), & 2 \pi-\varepsilon \leqslant \theta<2 \pi .\end{cases}
$$

Assume that $\varepsilon<\pi / 4$, to ensure that the patch is contained in the same horizontal strip as the arc which it replaces. Assume also that $\varepsilon$ is smaller than any $\left|1-\alpha_{i}\right|$ or $\left|1-\beta_{j}\right|$. We have

$$
|f(s)|<1, \quad s \in \Gamma .
$$

In other words, the image of $\Gamma$ under the mapping $f$ is contained in the open unit disc $|s|<1$. Its image under the mapping $1-f$ is thus contained in the domain $\operatorname{Re} s>0$, and has no windings about the origin. This can be written in the following form ${ }^{9}$ :

$$
\frac{1}{2 \pi} \Delta_{\Gamma} \arg \{1-f(s)\}=0 .
$$

In addition, $1-f$ has no zeros on $\Gamma$, by equation (16). Hence, $1-f$ has an equal number of zeros and poles inside $\Gamma$, by the argument principle. But the count of $\alpha_{i}$ 's inside the unit circle is one less than the total count of zeros of $1-f$ inside $\Gamma$, which includes $s=1$ as well.

\footnotetext{
${ }^{8}$ On the argument principle see, e.g., Nehari [10, p. 130].

${ }^{9} \mathrm{Cf}$. ibid.
} 


\section{Acknowledgement}

I am pleased to thank Professor J.W. Cohen for the inspiring discussions and for the spurring criticism.

\section{References}

[1] S. Asmussen, Applied Probability and Queues (Wiley, New York, 1987).

[2] S. Asmussen, Aspects of matrix Wiener-Hopf factorization in applied probability, Math. Scientist 14 (1989) 101-116.

[3] N. Bayer and O.J. Boxma, Wiener-Hopf analysis of an M/G/1 queue with negative customers and of a related class of random walks, Queueing Systems, this issue.

[4] A.A. Borovkov, Stochastic Processes in Queueing Theory (Springer-Verlag, 1976).

[5] J.W. Cohen, The Wiener-Hopf technique in applied probability, in: Perspectives in Probability and Statistics: Papers in Honour of M.S. Bartlett on the Occasion of His Sixty-Fifth Birthday, ed. J. Gani (Applied Probability Trust, 1975) pp. 145-156.

[6] J.H.A. de Smit, The single-server semi-Markov queue, Stochastic Processes and their Applications 22 (1986) 37-50.

[7] L. Kleinrock, Queueing Systems, Volume I: Theory (Wiley, New York, 1975).

[8] V.I. Lotov, On Some Boundary Crossing Problems for Gaussian Random Walks (Institute of Mathematics, Novosibirsk, October, 1994).

[9] V.I. Lotov and V.R. Khodjibayev, On the number of crossings of a strip for stochastic processes with independent increments, Siberian Advances in Mathematics 3(2) (1993) 145-152.

[10] Z. Nehari, Conformal Mapping (McGraw-Hill, New York, 1952). 